\title{
LPV Unknown Input Observer for Attitude of a mass-varying quadcopter
}

\author{
The Hung PHAM ${ }^{1}$ and Dalil ICHALAL ${ }^{2}$ and Said MAMMAR ${ }^{3}$
}

\begin{abstract}
In this paper, a Linear Parameter Varying (LPV) Unknown Input Observer (UIO) design for the attitude subsystem of a mass-varying quadcopter is proposed by using algebraic matrix manipulation. First, the design of UIO for LPV systems is discussed. Then, by applying the design process of UIO for the general LPV system, an LPV UIO is designed for the attitude of a mass-varying quadcopter. Simulation results in Matlab are conducted for illustrating the promising results of the paper.
\end{abstract}

\section{INTRODUCTION}

The problems of observing the state and the unknown inputs of a linear/non-linear dynamic system are difficult and challenging issues and have been studied since the 1970's [1][2][3]. An observer can play the aims of a virtual (software) sensor. This virtual sensor is aimed to estimate system parameters that are difficult or impossible to measure such as states or unknown inputs (faults, disturbances,...).

Several extensions for the observer of linear and nonlinear systems have been proposed and studied after the research proposed by D.G. Luenberger in [4]. The state estimation error is expressed as a system which is free from any known input in decoupling approach. The full rank conditions ensure the necessary and sufficient conditions for the existence of the observer [5][6]. For the second approach, both state vector and unknown input are estimated at the same time under the assumption that unknown inputs are part of the state vector [7][8].

In [9], a nonlinear disturbance observer-based backstepping controller is developed for attitude, altitude, and position control subject to some external disturbances. The stability analysis of the nonlinear disturbance observer is successfully done using the Lyapunov stability theory. In [10], a sliding mode control scheme is proposed for a quadrotor in the presence of an exogenous disturbance. The authors propose a disturbance observer to reject the effect of the unknown disturbance on the quadrotor by using a nonlinear sliding mode surface.

For a quadrotor, it is really important to determine the external forces and moments such as the force of the wind. However, forces and moments are really difficult to measure during the operation. Therefore, an alternative solution is

\footnotetext{
${ }^{1}$ The Hung PHAM is with IBISC, Evry-Val-d'Essonne University, Universite Paris-Saclay, Evry, France; and Le Quy Don Technical University, Hanoi, Viet Nam, thehung.pham@univ-evry. fr, thehungpham83@gmail.com.

${ }^{2}$ Dalil ICHALAL, and ${ }^{3}$ Said MAMMAR are with IBISC, EvryVal-d'Essonne University, Universite Paris-Saclay, Evry, France dalil.ichalal@univ-evry.fr, said.mammar@univevry.fr.
}

to use an observer. Furthermore, the mass and moments of inertia of UAVs are important constraints to take into account, however, for some reasons, the mass is varying. For example, in applications for spraying pesticides, the mass and moments of inertia of flying equipment will vary slowly over time. Meanwhile, with the job of transporting goods, the mass and moments of inertia of aircraft change abruptly. Mass change implies the changes of moments of inertia. Because of the changes of quadcopter's parameter (mass and moments of inertia), the fixed structure observer might not be working precisely. These issues can be addressed using LPV UI observers [11].

The aim of this paper is to design an LPV Unknown Input Observer for the attitude of a mass-varying quadcopter, which can estimate the external torques. The feature of this proposed LPV UIO is that its structure can vary along with the changes of the mass and moments of inertia. To begin with, the design of UIO for the LPV system is considered. The existence conditions of the LPV UI observer is studied, and then the gains of the UI observer are calculated by resolving LMIs, which ensure the convergence to zero of the state estimation error and the unknown input estimation error. Then, from the dynamic of the attitude of the massvarying quadcopter, the LPV altitude system is generated. Finally, an LPV UIO is designed for the altitude system of the mass-varying quadcopter.

The remainder of this paper is organized as follows: Section II presents the dynamical model of the quadcopter and some preliminary concepts for designing the Unknown Input LPV observer. Section III is dedicated to designing the LPV UIO for the attitude of a mass-varying quadcopter. Then, simulation results are presented in section IV. Finally, conclusions and some future work proposals in section $\mathrm{V}$ wrap up the paper.

\section{SySTEM MODEL AND PROBLEM STATEMENT}

\section{A. Problem formulation}

The aim of this section is to design an LPV UIO for a LPV system which structure is expressed by

$$
\left\{\begin{array}{l}
\dot{x}(t)=A(\rho(t)) x(t)+B(\rho(t)) u(t)+E(\rho(t)) d(t) \\
y(t)=C x(t)
\end{array}\right.
$$

where $x(t) \in R^{n}$ is the state vector, $u(t) \in R^{n_{u}}$ defines the input vector, $d(t) \in R^{d}$ represents the unknown input, and $y(t) \in R^{y}$ is the output of the system. $A(\cdot), B(\cdot)$, and $D(\cdot)$ are the varying parameters matrices with appropriate dimensions, while $C$ is a constant matrix. The time-varying vector $\rho^{T}(t)=\left(\rho_{1}, \ldots, \rho_{n_{\rho}}\right)$ contains $n_{\rho}$ varying parameters 
$\rho_{1}, \ldots, \rho_{n_{\rho}}$ which are assumed to be sufficiently smooth and bounded, in other words, $\rho(t)$ is an element of $\Theta$, a hyperrectangle defined by

$$
\Theta=\left\{\rho(t) \in R^{n_{\rho}} \mid \rho_{1} \in\left[\underline{\rho}_{1}, \bar{\rho}_{1}\right], \ldots, \rho_{n_{p}} \in\left[\underline{\rho}_{n_{p}}, \bar{\rho}_{n_{p}}\right]\right\}
$$

where $\rho_{i}$ and $\bar{\rho}_{i}, i=1, \ldots, n_{\rho}$ define the lower and upper bounds of the varying parameter $\rho_{i}(t)$.

For sake of simplicity, in what follows we put $\Delta(\rho(t))$ as $\Delta_{\rho}$ where $\Delta$ is a matrix depending on the varying parameter $\rho(t)$. In addition, for the purpose of eliminating the input from the system (1), we define an auxiliary system of the form

$$
\left\{\begin{aligned}
\dot{s}(t) & =A_{\rho} s(t)+B_{\rho} u(t) \\
y_{s}(t) & =C s(t)
\end{aligned}\right.
$$

Define the errors $z(t)=x(t)-s(t)$ and $y_{z}(t)=y(t)-$ $y_{s}(t)$, the new dynamics

$$
\left\{\begin{aligned}
\dot{z}(t) & =A_{\rho} z(t)+E_{\rho} d(t) \\
y_{z}(t) & =C z(t)
\end{aligned}\right.
$$

can be obtained from the systems in (1) and (3). We can see that the new dynamics system in (4) are free from the known input $u(t)$. As a result, after estimating the new state $\hat{z}(t)$, we obtain the real state $\hat{x}(t)$ with the equation

$$
\hat{x}(t)=\hat{z}(t)+s(t)
$$

Consequently, the problem of designing the LPV UIO for LPV system in (1) is transformed to the problem of designing the LPV UIO for the LPV system without the known input depicted in (4).

Definition 1: If there exists positive integers $k_{y}, k_{u}$ and $k_{\rho}$ such that $\rho^{(j)}(t) \in \Theta_{j}$ for $j=0, \ldots, k_{\rho}$, and the state of the dynamics system in (1) can be expressed as a vector function which contains the system outputs, system inputs, the varying parameters, and their time derivatives up to a finite order as the following equation

$$
x(t)=F\left(y(t), \ldots, y^{\left(k_{y}\right)}, u(t), \ldots, u^{\left(k_{u}\right)}, \rho(t), \ldots, \rho^{\left(k_{\rho}\right)}\right)
$$

Then the system (1) is called uniformly strongly algebraically observable [11] with respect to the varying parameter $\rho(t)$.

We also assume that the time derivatives of the varying parameters belong to the compact sets defined by

$\Theta_{j}=\left\{\rho^{(j)} \in R^{n_{\rho}} \mid \rho_{1}^{(j)} \in\left[\underline{\rho}_{1 j}, \bar{\rho}_{1 j}\right], \ldots, \rho_{n_{p}}^{(j)} \in\left[\underline{\rho}_{n_{p} j}, \bar{\rho}_{n_{p} j}\right]\right\}$

where $\underline{\rho}_{i j}$ is the the lower and $\bar{\rho}_{i j}, i=1, \ldots, n_{\rho}$ is the upper bound of the $j$ th derivative of the varying parameter $\rho_{i}(t)$.

Definition 2: Suppose we have a dynamics system as shown in (1). If the unknown input $d(t)$ of the system is appeared in the equation of the $r^{t h}$ time derivative of the output $\left(y^{(r)}(t)\right)$, where $r$ is a non-negative integer number, then $r$ is called the relative degree [12] of the output $y(t)$ with respect to the unknown input $d(t)$.
1) LPV UIO design for LPV system (The following results are summarized and rewritten from [11]): Let us consider the LPV system in (4) where $y(t) \in R^{n_{y}}$ and $d(t) \in R^{n_{d}}$. Suppose that, each output $y_{i}(t)$ has a relative degree $r_{i}$ where $i=1, \ldots, n_{y}$ with respect to the unknown inputs. Thus, the vector relative order is given by $\left\{r_{1}, r_{2}, \ldots, r_{n_{y}}\right\}$.

The matrices $E_{\rho}(\rho(t))$ and $C$ can be rewritten as $E_{\rho}(\rho(t))=\left[\begin{array}{llll}E_{\rho}^{1} & E_{\rho}^{2} & \ldots & E_{\rho}^{n_{d}}\end{array}\right], C=\left[\begin{array}{lll}C_{1} & \ldots & C_{n_{y}}\end{array}\right]^{T}$

By differentiating $r_{i}$ times the $i^{t h}$ output $y_{i}(t)$, ones gets

$$
\begin{aligned}
y_{i}(t) & =C_{i} x(t) \\
\dot{y}_{i}(t) & =\underbrace{C_{i} A_{\rho}}_{M_{1 \rho}^{i}} x(t) \\
\ddot{y}_{i}(t) & =\underbrace{\left(M_{1 \rho}^{i} A_{\rho}+\dot{M}_{1 \rho}^{i}\right)}_{M_{2 \rho}^{i}} x(t) \\
y_{i}^{(3)}(t)= & \underbrace{\left(M_{2 \rho}^{i} A_{\rho}+\dot{M}_{2 \rho}^{i}\right)}_{M_{3 \rho}^{i}} x(t) \\
y_{i}^{\left(r_{i}\right)}(t)= & \underbrace{\left(M_{\left(r_{i}-1\right) \rho}^{i} A_{\rho}+\dot{M}_{\left(r_{i}-1\right) \rho}^{i}\right)}_{M_{r_{i} \rho}^{i}} x(t) \\
& +M_{\left(r_{i}-1\right) \rho}^{i} E_{\rho}^{i} d_{i}(t)
\end{aligned}
$$

From (8a) to (8e), the output time derivatives can be expressed in the matrix form as following

$$
\mathscr{Y}(t)=\mathscr{M}_{\rho} z(t)+\Gamma_{\rho} d(t)
$$

where

$$
\mathscr{Y}(t)=\left[\begin{array}{c}
y_{1}^{\left(r_{1}\right)}(t) \\
y_{2}^{\left(r_{2}\right)}(t) \\
\vdots \\
y_{n_{y}}^{\left(r_{n_{y}}\right)}(t)
\end{array}\right], \mathscr{M}_{\rho}(t)=\left[\begin{array}{c}
M_{r_{1} \rho}^{1} \\
M_{r_{2} \rho}^{2} \\
\vdots \\
M_{r_{n y} \rho}^{n_{y}}
\end{array}\right]
$$

and

$$
\Gamma_{\rho}=\left[\begin{array}{cccc}
M_{\left(r_{1}-1\right)}^{1} E_{\rho}^{1} & M_{\left(r_{1}-1\right)}^{1} E_{\rho}^{2} & \ldots & M_{\left(r_{1}-1\right)}^{1} E_{\rho}^{n_{d}} \\
M_{\left(r_{2}-1\right)}^{2} E_{\rho}^{1} & M_{\left(r_{2}-1\right)}^{2} E_{\rho}^{2} & \ldots & M_{\left(r_{2}-1\right)}^{2} E_{\rho}^{n_{d}} \\
\vdots & \vdots & \ddots & \vdots \\
M_{\left(r_{n}-1\right)}^{n_{y}} E_{\rho}^{1} & M_{\left(r_{n y}-1\right)}^{n_{y}} E_{\rho}^{2} & \ldots & M_{\left(r_{n}-1\right)}^{n_{y}} E_{\rho}^{n_{d}}
\end{array}\right]
$$

Consequently, the observer for (4) is proposed in the form

$$
\left\{\begin{array}{l}
\dot{\hat{z}}(t)=\left(A_{\rho}-Q_{\rho} \mathscr{M}_{\rho}-L_{\rho} C\right) \hat{z}(t)+Q_{\rho} \mathscr{Y}(t)+L_{\rho} y_{z}(t) \\
\hat{d}(t)=\Gamma_{\rho}^{-1}\left(\mathscr{Y}(t)-\mathscr{M}_{\rho} \hat{z}(t)\right)
\end{array}\right.
$$

where $Q_{\rho}$ and $L_{\rho}$ are to be determined.

Theorem 1: The dynamics system (10) is called an observer for the dynsmics system (4) if the following conditions hold:

- The matrix $\Gamma$ is full column rank 
- The pair $\left(A_{\rho}-Q_{\rho} \mathscr{M}_{\rho}, C\right)$ is detectable $\forall \rho \in \Theta$, where $Q_{\rho}=E \Gamma^{-1}$ if $n_{y}=n_{d}$, and $Q_{\rho}=E \Gamma^{\dagger}$ if $n_{y}>n_{d}, \Gamma^{\dagger}$ is the pseudo inverse of $\Gamma$

- The parameter varying matrix $\left(A_{\rho}-Q_{\rho} \mathscr{M}_{\rho}-L_{\rho} C\right)$ is stable $\forall \rho \in \Theta$

Proof: The state estimation error is defined as $e(t)=$ $z(t)-\hat{z}(t)$ and the unknown input estimation error $e_{d}(t)=$ $d(t)-\hat{d}(t)$. The derivative of the error is

$$
\begin{aligned}
\dot{e}_{z}(t)= & \dot{z}(t)-\dot{z}(t) \\
= & A_{\rho} z(t)+E_{\rho} d(t)-\left(A_{\rho}-Q_{\rho} \mathscr{M}_{\rho}-L_{\rho} C\right) \hat{z}(t) \\
& -Q_{\rho} \mathscr{Y}(t)-L_{\rho} y_{z}(t) \\
= & \left(A_{\rho}-Q_{\rho} \mathscr{M}_{\rho}-L_{\rho} C\right) e(t)+\left(E_{\rho}-Q_{\rho} \Gamma_{\rho}\right) d(t)
\end{aligned}
$$

Under the condition that the matrix $\Gamma$ is full column rank, $\Gamma^{-1}\left(\Gamma^{\dagger}\right)$ exists and ensures that $E_{\rho}-Q_{\rho} \Gamma_{\rho}=0$, the state estimation dynamics becomes

$$
\dot{e}_{z}(t)=\left(A_{\rho}-L_{\rho} C-Q_{\rho} \mathscr{M}_{\rho}\right) e(t)
$$

When the pair $\left(A_{\rho}-Q_{\rho} \mathscr{M}_{\rho}, C\right)$ is detectable for $\forall \rho \in$ $\Theta$, we can determine the gain matrix $L_{\rho}$ for ensuring the asymptotic stability of the system (12). Thus, the state of the observer is ensured to converge asymptotically to the state of the system. Consequently, the unknown input estimation

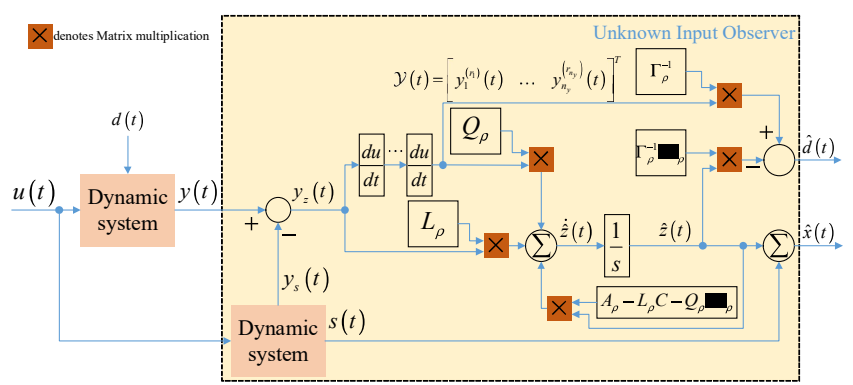

Fig. 1. Unknown Input Observer for dynamic system

can be determined as following

$$
e_{d}(t)=d(t)-\hat{d}(t)=-\Gamma_{\rho}^{-1} \mathscr{M}_{\rho} e_{z}(t)
$$

which ensures that $e_{d}$ converges towards 0 .

The structure of UIO in (10) is depicted in figure 1.

2) Convergence analysis and LMI formulation: From (12) and (13), we have

$$
\left\{\begin{array}{l}
\dot{e}_{z}(t)=\left(A_{\rho}-Q_{\rho} \mathscr{M}_{\rho}-L_{\rho} C\right) e_{z}(t) \\
e_{d}(t)=-\Gamma_{\rho}^{-1} \mathscr{M}_{\rho} e_{z}(t)
\end{array}\right.
$$

We next transform the matrices $A_{\rho}, Q_{\rho}, \mathscr{M}_{\rho}$, and $\Gamma_{\rho}$ in a polytopic form where the parameters $\rho(t) \in \Theta$, one obtains

$$
\left\{\begin{aligned}
A_{\rho} & =\sum_{i=1}^{2^{n} \rho} \mu_{i}(\rho(t)) A_{i} \\
Q_{\rho} & =\sum_{i=1}^{2^{n} \rho} \mu_{i}(\rho(t)) Q_{i} \\
\mathscr{M}_{\rho} & =\sum_{i=1}^{2^{n} \rho} \mu_{i}(\rho(t)) \mathscr{M}_{i} \\
\Gamma_{\rho} & =\sum_{i=1}^{2^{n} \rho} \mu_{i}(\rho(t)) \Gamma_{i}
\end{aligned}\right.
$$

$\forall \rho(t) \in \Theta$ and $\mu_{i}(\rho(t))$ satisfy the convex sum property

$\sum_{i=1}^{2^{n} \rho} \mu_{i}(\rho(t))=1, \quad 0 \leq \mu_{i}(\rho(t)) \leq 1, \quad i=1, \ldots 2^{n_{\rho}}, \quad \forall \rho(t) \in \Theta$

Thus, the gain matrix $L_{\rho}$ can be determined as

$$
L_{\rho}=\sum_{i=1}^{2^{n} \rho} \mu_{i}(\rho(t)) L_{i}
$$

Thus, the state estimation error dynamics as shown in (12) can be rewritten as follows

$$
\dot{e}_{z}(t)=\sum_{i=1}^{2^{n} \rho} \sum_{j=1}^{2^{n} \rho} \mu_{i}(\rho(t)) \mu_{j}(\rho(t))\left(A_{j}-Q_{i} \mathscr{M}_{j}-L_{j} C\right) e_{z}(t)
$$

Standard LMI for stability can be obtained using a common quadratic Lyapunov function in the form

$$
V\left(e_{z}(t)\right)=e_{z}^{T}(t) X e_{z}(t), X=X^{T}>0
$$

The procedure can be extended to polyquadratic Lyapunov functions. The derivative of the Lyapunov function is

$$
\dot{V}\left(e_{z}(t)\right)=\dot{e}_{z}^{T}(t) X e_{z}(t)+e_{z}^{T}(t) X \dot{e}_{z}(t)
$$

Finally, the derivative of the Lyapunov function $V\left(e_{z}(t)\right)$ in (20) is rewritten as follows

$$
\begin{gathered}
\dot{V}\left(e_{z}(t)\right)=e_{z}^{T}(t)\left[A_{\rho} X+X A_{\rho}-\left(Q_{\rho} M_{\rho}\right)^{T} X+X Q_{\rho} M_{\rho}\right. \\
\left.-C^{T} K_{\rho}^{T}-K_{\rho} C\right] e_{z}(t)
\end{gathered}
$$

where $K_{\rho}=L_{\rho} C$.

Using the time derivative of the Lyapunov function $V\left(e_{z}(t)\right)$, the state estimation error dynamics (18), and the convex sum property of the weighting functions in (16), we can determine the sufficient LMI conditions which ensure the asymptotic stability as in the follows equation

$$
\begin{aligned}
& A_{j}^{T} X+X A_{j}-\left(Q_{i} M_{j}\right)^{T} X+X Q_{i} M_{j}-C^{T} K_{j}^{T}-K_{j} C<0 \\
& i, j=1 \ldots 2^{n_{p}}
\end{aligned}
$$

The observer gains $L_{i}$ of the proposed unknown input observer are obtained as

$$
L_{i}=X^{-1} K_{i}
$$

The gathered observer $L_{\rho}$ as in (17) with observer gains $L_{i}$ in (23) ensures that the state estimation error $e(t)$ and the unknown input estimation error $e_{d}(t)$ convergence to zero. 


\section{B. Quadrotor model}

The objective of this paper is developing an LPV UI observer for the attitude subsystem of a quadcopter. The quadcopter has six degrees of freedom and only four actuators. It is thus under-actuated. A quadcopter is a helicopter

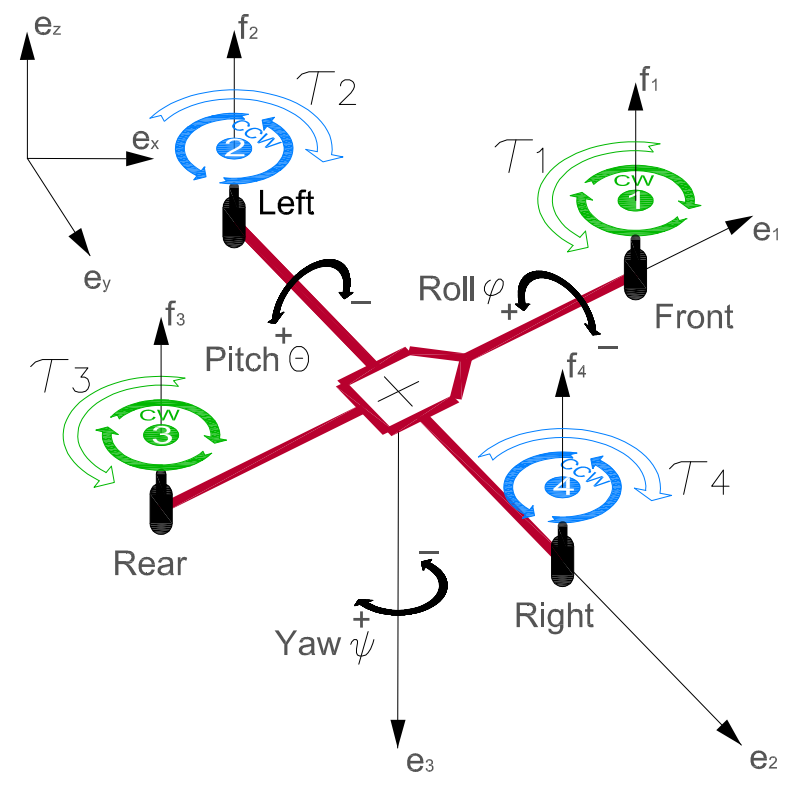

Fig. 2. Quadcopter

that consists of a rigid cross-frame equipped with four rotors as shown in Fig. 2. Its four rotors generate four independent thrusts. In order to avoid the yaw drift due to the reactive torques, the quadrotor aircraft is configured such that the set of rotors $M_{2}, M_{4}$ (left-right) revolves at angular speeds $\omega_{1}$ and $\omega_{2}$ in clockwise (CW) direction generating thrusts of $\tau_{1}$ and $\tau_{3}$, while the pair of rotors $M_{1}, M_{3}$ (front-rear) rotates at angular speeds $\omega_{2}$ and $\omega_{4}$ in counterclockwise (CCW) direction generating thrusts of $\tau_{2}$ and $\tau_{4}$. The direction of rotation of the rotors are fixed (i.e., $\omega_{i} \geq 0, i \in\{1,2,3,4\}$ ). The forward/backward left/right, and the yaw motions are achieved through a differential control strategy of the thrust generated by each rotor.

Let $I=\left\{e_{x}, e_{y}, e_{z}\right\}$ denotes the inertial frame, and $A=$ $\left\{e_{1}, e_{2}, e_{3}\right\}$ denote the frame rigidly attached to the quadrotor as shown in Fig. 2.

The mathematical model of the quadcopter was generated by the techniques of both Euler-Newton [13] and EulerLagrange [14], given as follows:

$$
\left\{\begin{aligned}
\ddot{x} & =(\sin \psi \sin \varphi+\cos \psi \sin \theta \cos \varphi) \frac{U_{1}}{m} \\
\ddot{y} & =(\sin \psi \sin \theta \cos \varphi-\cos \psi \sin \varphi) \frac{U_{1}}{m} \\
\ddot{z} & =(\cos \theta \cos \varphi) \frac{U_{1}}{m}-g \\
\ddot{\varphi} & =\frac{I_{y}-I_{z}}{I_{x}} \dot{\theta} \dot{\psi}-\frac{J_{r} \Omega_{r}}{I_{x}} \dot{\theta}+\frac{l}{I_{x}} U_{2} \\
\ddot{\theta} & =\frac{I_{z}-I_{x}}{I_{y}} \dot{\varphi} \dot{\psi}+\frac{J_{r} \Omega_{r}}{I_{y}} \dot{\varphi}+\frac{l}{I_{y}} U_{3} \\
\ddot{\psi} & =\frac{I_{x}-I_{y}}{I_{z}} \dot{\varphi} \dot{\theta}+\frac{1}{I_{z}} U_{4}
\end{aligned}\right.
$$

where $m$ denotes the mass the of the quadcopter, $(x, y, z)$ are the three positions, $(\varphi, \theta, \psi)$ are the three Euler angles, $I_{x}$,
$I_{y}$, and $I_{z}$ are the moments of inertia w.r.t the three axes $x, y$, and $z$ respectively; $J_{r}$ is the moment of inertia of the rotors, $l$ represents the distance from the rotors to the center of mass of the quadcopter aircraft. $\Omega_{r}$ is the overall residual propeller angular speed, $b$ and $d$ are thrust and drag coefficients. The quadcopter's inputs are: the thrust force $\left(U_{1}\right)$ and three torques (roll torque $\left(U_{2}\right)$, pitch torque $\left(U_{3}\right)$, and yaw torque $\left.\left(U_{4}\right)\right)$, the force and torques are related on the rotor speed as follows:

$$
\left\{\begin{array}{l}
U_{1}=b\left(\omega_{1}^{2}+\omega_{2}^{2}+\omega_{3}^{2}+\omega_{4}^{2}\right) \\
U_{2}=b\left(\omega_{4}^{2}-\omega_{2}^{2}\right) \\
U_{3}=b\left(\omega_{3}^{2}-\omega_{1}^{2}\right) \\
U_{4}=d\left(\omega_{1}^{2}-\omega_{2}^{2}+\omega_{3}^{2}-\omega_{4}^{2}\right)
\end{array}\right.
$$

and

$$
\Omega_{r}=\omega_{1}-\omega_{2}+\omega_{3}-\omega_{4}
$$

The first three equations of the system of differential equations in (24) denote the transnational movement, while the last three present the rotational movement of the quadcopter. We restrict the purpose of the paper to design a UIO for the attitude subsystem of a quadcopter. Thus, the equations related to the longitudinal, lateral, and altitude motions of the quadcopter are removed.

Remark 1: Suppose that $\mathrm{n}$ objects $o_{1}, \ldots, o_{n}$ are attached to the quadcopter, and the mass of quadcopter and objects are $m_{q}, m_{o_{1}}, \ldots, m_{o_{n}}$ respectively. Therefore, the mass of the system consists of the quadcopter and $n$ objects can be easily calculated by the equation $m=m_{q}+m_{o_{1}}+\ldots+m_{o_{n}}$. When the object $o_{i}$ is detached from the quadcopter for $i=n, \ldots, 1$, the remaining mass of the system can be recalculated.

Depending on the mass and shape of each object, one can calculate its moments of inertia around the axes passing through its center of mass. When attaching these objects to the quadcopter, based on their shapes and positions with respect to the center of gravity $G$ of the quadcopter, their moments of inertia with respect to the three axes $I_{x}, I_{y}, I_{z}$ of the quadcopter can be calculated. Thus the moment of inertia of the system which contains quadcopter and $n$ objects $o_{1}, \ldots, o_{n}$ relative to $I_{x}, I_{y}, I_{z}$ can be calculated.

Another online approach to estimate the geometric and inertia parameters of a multi-rotor aerial vehicle is developed in [15].

Remark 2: Suppose that each actuator thrust Laplace transform is given by

$$
\mathscr{T}_{i}(s)=\frac{K_{i}}{1+\kappa_{i} s} V_{i}(s), \quad i=1,2,3,4
$$

where $\mathscr{T}_{i}$ is the Laplace transform of the thrust $T_{i}(t), V_{i}$ is the pulse width modulation (PWM) voltage, $K_{i}$ is the armature gain, and $\kappa_{i}$ is the time constant of the $i^{t h}, i=1, \ldots, 4$ rotor. The corresponding differential equation is

$$
\dot{T}_{i}=-\frac{1}{\kappa_{i}} T_{i}+\frac{K_{i}}{\kappa_{i}} v_{i}
$$

We also known that, the thrust $T_{i}(t)$ is a function of the rotor speed

$$
T_{i}(t)=k_{f} \Omega_{i}^{2}
$$


where $k_{f}$ is the constant coefficient.

Consequently, based on the PWM applied to each rotor, the rotor speed $\omega_{i}$ can be estimated. Thus, the residual speed $\Omega_{r}=\omega_{2}+\omega_{4}-\omega_{1}-\omega_{3}$ can also be estimated.

\section{LPV UNKNOWN INPUT OBSERVER DESIGN}

The proposed LPV Unknown input observer based on Linear Matrix Inequalities methods, the resolvability of the resulting LMI conditions in this case is quite compromised due to conservativeness of conditions which will request the common stabilization of a huge number of submodels. In order to reduce this number, we adopt here a more simplified model. We assume that the quadcopter is symmetric and $I_{x}=I_{y}$. We also assume that to the altittude sub-model of the quadcopter is disturbed the torques $d(t)=\left[\begin{array}{lll}d_{\varphi} & d_{\theta} & d_{\psi}\end{array}\right]^{T}$ allowing to write:

$$
\left\{\begin{aligned}
\ddot{\varphi} & =\frac{I_{y}-I_{z}}{I_{x}} \dot{\theta} \dot{\psi}-\frac{J_{r} \Omega_{r}}{I_{x}} \dot{\theta}+\frac{l}{I_{x}} U_{2}+\frac{1}{I_{x}} d_{\varphi} \\
\ddot{\theta} & =\frac{I_{z} I_{x}}{I_{x}} \dot{\varphi} \dot{\psi} \frac{J_{r} \Omega_{r}}{I_{x}} \dot{\varphi}+\frac{l}{I_{x}} U_{3}+\frac{1}{I_{x}} d_{\theta} \\
\ddot{\psi} & =\frac{1}{I_{z}} U_{4}+\frac{1}{I_{z}} d_{\psi}
\end{aligned}\right.
$$

We can see that, the dynamic equation of the yaw angle does not contain Euler angles or their derivatives. Thus, the dynamic of quadcopter attitude can be decomposed into two subsystems, the roll-pitch subsystem is the first two equations and the yaw subsystem is the third equation of (30).

\section{A. LPV UIO for Roll-Pitch}

The system differential equations for $\varphi$ and $\theta$ are rewritten from (30) as

$$
\left\{\begin{aligned}
\dot{\varphi} & =\dot{\varphi} \\
\dot{\theta} & =\dot{\theta} \\
\ddot{\varphi} & =\frac{I_{y}-I_{z}}{I_{x}} \dot{\theta} \dot{\psi}-\frac{J_{r} \Omega_{r}}{I_{x}} \dot{\theta}+\frac{l}{I_{x}} U_{2}+\frac{1}{I_{x}} d_{\varphi} \\
\ddot{\theta} & =\frac{I_{z}-I_{x}}{I_{x}} \dot{\varphi} \dot{\psi}+\frac{J_{r} \Omega_{r}}{I_{x}} \dot{\varphi}+\frac{l}{I_{x}} U_{3}+\frac{1}{I_{x}} d_{\theta}
\end{aligned}\right.
$$

LPV system for roll pitch can be obtained from system differential equation (31) as

$$
\left\{\begin{array}{l}
\dot{x}_{1}(t)=A_{1_{\rho_{1}}} x_{1}(t)+B_{1_{\rho_{1}}} u_{1}(t)+E_{1_{\rho_{1}}} d_{1}(t) \\
y_{1}(t)=C_{1} x_{1}(t)
\end{array}\right.
$$

where varying parameters, state, output, known input, and unknown input are respectively $\rho_{1}(t)=\left[\begin{array}{ll}\rho_{11} & \rho_{12}\end{array}\right]^{T}=\left[\begin{array}{cc}\frac{1}{I_{x}} & \frac{I_{y}-I_{z}}{I_{x}} \psi-\frac{J_{r} \Omega_{r}}{I_{x}}\end{array}\right]^{T}$, $x_{1}(t)=\left[\begin{array}{llll}\varphi & \theta & \dot{\varphi} & \dot{\theta}\end{array}\right]^{T} ; y_{1}(t)=\left[\begin{array}{cccc}\varphi & \theta & \dot{\varphi} & \dot{\theta}\end{array}\right]^{T}$, $u_{1}(t)=\left[\begin{array}{ll}U_{2} & U_{3}\end{array}\right] ; d_{1}(t)=\left[\begin{array}{ll}d_{\varphi} & d_{\theta}\end{array}\right]^{T}$ and the system matrices are:

$$
\begin{aligned}
& A_{1_{\rho_{1}}}=\left[\begin{array}{cccc}
0 & 0 & 1 & 0 \\
0 & 0 & 0 & 1 \\
0 & 0 & 0 & \rho_{12} \\
0 & 0 & -\rho_{12} & 0
\end{array}\right] ; B_{1_{\rho_{1}}}=\left[\begin{array}{cc}
0 & 0 \\
0 & 0 \\
l \rho_{11} & 0 \\
0 & l \rho_{11}
\end{array}\right] \\
& C_{1}=\left[\begin{array}{cccc}
1 & 0 & 0 & 0 \\
0 & 1 & 0 & 0 \\
0 & 0 & 1 & 0 \\
0 & 0 & 0 & 1
\end{array}\right] ; E_{1_{\rho_{1}}}=\left[\begin{array}{cc}
0 & 0 \\
0 & 0 \\
\rho_{11} & 0 \\
0 & \rho_{11}
\end{array}\right]
\end{aligned}
$$

Define an auxiliary system in the form

$$
\left\{\begin{aligned}
\dot{s}_{1}(t) & =A_{1_{\rho_{1}}} s_{1}(t)+B_{1_{\rho_{1}}} u_{1}(t) \\
y_{s_{1}}(t) & =C_{1} s_{1}(t)
\end{aligned}\right.
$$

Define the errors $z_{1}(t)=x_{1}(t)-s_{1}(t)$ and $y_{z_{1}}(t)=y_{1}(t)-$ $y_{s_{1}}(t)$, the new dynamics

$$
\left\{\begin{aligned}
\dot{z}_{1}(t) & =A_{1_{\rho_{1}}} z_{1}(t)+E_{1_{\rho_{1}}} d_{1}(t) \\
y_{z_{1}}(t) & =C_{1} z_{1}(t)
\end{aligned}\right.
$$

can be obtained from the systems in (32) and (34). We can see that the new dynamics system in (35) are free from the known input $u_{1}(t)$. As a result, after estimating the new state $\hat{z}_{1}(t)$, we obtain the real state $\hat{x}_{1}(t)$ with the equation $\hat{x}_{1}(t)=$ $\hat{z}_{1}(t)+s_{1}(t)$.

Take the derivative of the output $y_{z_{1}}=$ $\left[\begin{array}{llll}y_{z_{11}} & y_{z_{12}} & y_{z_{13}} & y_{z_{14}}\end{array}\right]^{T}$ as in (8a) to (8e), one obtains:

$$
\left\{\begin{array}{l}
y_{z_{11}}^{(2)}=\rho_{12} \tau_{4}+\rho_{11} d_{\varphi} \\
y_{z_{12}}^{(2)}=-\rho_{12} \tau_{3}+\rho_{11} d_{\theta} \\
y_{z_{13}}^{(1)}=\rho_{12} \tau_{4}+\rho_{11} d_{\varphi} \\
y_{z_{14}}^{(1)}=-\rho_{12} \tau_{3}+\rho_{11} d_{\theta}
\end{array}\right.
$$

Consequently, the relative degrees $r_{i}$ of the $i^{\text {th }}$ output, $i=$ $1, . ., 4$ respectively are $r_{11}=2, r_{12}=2, r_{13}=1$, and $r_{14}=1$.

Following the defined vector $\mathscr{Y}_{1}(t)=$ $\left[\begin{array}{llll}y_{z_{11}}^{(2)} & y_{z_{12}}^{(2)} & y_{z_{13}}^{(1)} & y_{z_{14}}^{(1)}\end{array}\right]^{T}=\mathscr{M}_{1_{\rho_{1}}} z_{1}(t)+\Gamma_{1_{\rho_{1}}} d_{1}(t)$ in (9), one obtains

$$
\Gamma_{1_{\rho_{1}}}=\left[\begin{array}{cc}
\rho_{11} & 0 \\
0 & \rho_{11} \\
\rho_{11} & 0 \\
0 & \rho_{11}
\end{array}\right]
$$

which satisfies the full column rank condition and its pseudoinverse is given by

$$
\Gamma_{1_{\rho_{1}}}^{\dagger}=\left[\begin{array}{cccc}
\frac{1}{2 \rho_{11}} & 0 & \frac{1}{2 \rho_{11}} & 0 \\
0 & \frac{1}{2 \rho_{11}} & 0 & \frac{1}{2 \rho_{11}}
\end{array}\right]
$$

The unknown input decoupling matrix $Q_{1_{\rho_{1}}}$ is then given by

$$
Q_{\rho_{\rho_{1}}}=\left[\begin{array}{cccc}
0 & 0 & 0 & 0 \\
0 & 0 & 0 & 0 \\
\frac{1}{2} & 0 & \frac{1}{2} & 0 \\
0 & \frac{1}{2} & 0 & \frac{1}{2}
\end{array}\right]
$$

We can verify that the pair $\left(A_{1_{\rho_{1}}}-Q_{1_{\rho_{1}}} \mathscr{M}_{1_{\rho_{1}}}, C_{1}\right)$ is detectable, where the matrix $\mathscr{M}_{\rho_{\rho_{1}}}$ and $A_{1_{\rho_{1}}}-Q_{1_{\rho_{1}}} \mathscr{M}_{\rho_{\rho_{1}}}$ are respectively

$$
\begin{gathered}
\mathscr{M}_{\rho_{\rho_{1}}}=\left[\begin{array}{cccc}
0 & 0 & 0 & \rho_{12} \\
0 & 0 & -\rho_{12} & 0 \\
0 & 0 & 0 & \rho_{12} \\
0 & 0 & -\rho_{12} & 0
\end{array}\right] \\
A_{1_{\rho_{1}}}-Q_{1_{\rho}} \mathscr{M}_{1_{\rho_{1}}}=\left[\begin{array}{cc}
0_{2} & I_{2} \\
0_{2} & 0_{2}
\end{array}\right]
\end{gathered}
$$

Finally, the gain matrix $L_{1_{\rho}}$ of the UIO can be calculated such that the parameter varying matrix $A_{1_{\rho_{1}}}-Q_{1_{\rho_{1}}} \mathscr{M}_{1_{\rho_{1}}}-L_{1_{\rho_{1}}} C_{2}$ 
is stable $\forall \rho_{11}, \rho_{12} \in \Theta_{\rho_{1}}$ by solving LMIs as in (22), where $\Theta_{\rho_{1}}$ is the hyper rectangle for $\rho_{11}$ and $\rho_{12}$ which is defined in (2).

\section{B. LPV UIO for Yaw}

The system differential equations for $\psi$ are rewritten from (30) as

$$
\left\{\begin{array}{l}
\dot{\psi}=\dot{\psi} \\
\ddot{\psi}=\frac{1}{I_{z}} U_{4}+\frac{1}{I_{z}} d_{\psi}
\end{array}\right.
$$

LPV system for roll pitch can be obtained from system differential equation (41) as

$$
\left\{\begin{array}{l}
\dot{x}_{2}(t)=A_{2 \rho_{2}} x_{2}(t)+B_{2 \rho_{2}} u_{2}(t)+E_{2 \rho_{2}} d_{2}(t) \\
y_{2}(t)=C_{2} x_{2}(t)
\end{array}\right.
$$

where varying parameters, state, output, known input, and unknown input are respectively $\rho_{2}(t)=\left[\rho_{21}\right]=\left[\frac{1}{I_{z}}\right] ; x_{2}(t)=$ $\left[\begin{array}{cc}\psi & \dot{\psi}\end{array}\right]^{T} ; y_{2}(t)=\left[\begin{array}{cc}\psi & \dot{\psi}\end{array}\right]^{T} ; u_{2}(t)=\left[U_{4}\right] ; d_{2}(t)=\left[d_{\psi}\right]$ and the system matrices are

$$
A_{2 \rho_{2}}=\left[\begin{array}{cc}
0 & 0 \\
0 & 0
\end{array}\right] ; B_{2 \rho_{2}}=E_{2 \rho_{2}}=\left[\begin{array}{c}
0 \\
\rho_{21}
\end{array}\right] ; C_{2}=\left[\begin{array}{cc}
1 & 0 \\
0 & 1
\end{array}\right]
$$

Define an auxiliary system in the form

$$
\left\{\begin{aligned}
\dot{s}_{2}(t) & =A_{2 \rho_{2}} s_{2}(t)+B_{2 \rho_{2}} u_{2}(t) \\
y_{s_{2}}(t) & =C_{2} s_{2}(t)
\end{aligned}\right.
$$

Define the errors $z_{2}(t)=x_{2}(t)-s_{2}(t)$ and $y_{z_{2}}(t)=y_{2}(t)-$ $y_{s_{2}}(t)$, the new dynamics

$$
\left\{\begin{aligned}
\dot{z}_{2}(t) & =A_{2 \rho_{2}} z_{2}(t)+E_{2 \rho_{2}} d(t) \\
y_{z_{2}}(t) & =C_{2} z_{2}(t)
\end{aligned}\right.
$$

can be obtained from the systems in (42) and (44), We can see that the new dynamics system in (45) are free from the known input $u_{2}(t)$. As a result, after estimating the new state $\hat{z}_{2}(t)$, we obtain the real state $\hat{x}_{2}(t)$ with the equation $\hat{x}_{2}(t)=$ $\hat{z}_{2}(t)+s_{2}(t)$.

Take the derivative of the output $y_{z_{2}}=\left[\begin{array}{ll}y_{z_{21}} & y_{z_{22}}\end{array}\right]^{T}$ as in (8a) to (8e), one obtains:

$$
\left\{\begin{array}{l}
y_{z_{21}}^{(2)}=\rho_{21} d_{\psi} \\
y_{z_{21}}^{(1)}=\rho_{21} d_{\psi}
\end{array}\right.
$$

Consequently, the relative degrees $r_{i}$ of the $i^{\text {th }}, i=1,2$ output respectively are $r_{21}=2, r_{22}=1$.

Following the defined vector $\mathscr{Y}_{2}=\left[\begin{array}{ll}y_{z_{21}}^{(2)} & y_{z_{22}}^{(1)}\end{array}\right]^{T}$ in (9), one obtains

$$
\Gamma_{\rho_{2}}=\left[\begin{array}{l}
\rho_{21} \\
\rho_{21}
\end{array}\right]
$$

which satisfies the full column rank condition and the pseudo-inverse exists and given by

$$
\Gamma_{2 \rho_{1}}^{\dagger}=\left[\begin{array}{ll}
\frac{1}{2 \rho_{21}} & \frac{1}{2 \rho_{21}}
\end{array}\right]
$$

The unknown input decoupling matrix $Q_{1_{\rho}}$ is then given by

$$
Q_{2 \rho_{2}}=\left[\begin{array}{cc}
0 & 0 \\
\frac{1}{2} & \frac{1}{2}
\end{array}\right]
$$

We can verify that the pair $\left(A_{2_{\rho_{2}}}-Q_{2} \rho_{2} \mathscr{M}_{2 \rho_{2}}, C_{2}\right)$ is detectable, where the matrix $\mathscr{M}_{2_{\rho_{2}}}$ and $A_{\rho_{\rho_{2}}}-Q_{2_{\rho_{2}}} \mathscr{M}_{2_{\rho_{2}}}$ are respectively

$$
\mathscr{M}_{2_{\rho_{2}}}=\left[\begin{array}{ll}
0 & 0 \\
0 & 0
\end{array}\right] ; A_{2_{\rho_{2}}}-Q_{2_{\rho_{2}}} \mathscr{M}_{2_{\rho_{2}}}=\left[\begin{array}{ll}
0 & 1 \\
0 & 0
\end{array}\right]
$$

Finally, the gain matrix $L_{2 \rho_{2}}$ of the UIO can be calculated such that the parameter varying matrix $A_{2 \rho_{2}}-Q_{2_{\rho_{2}}} \mathscr{M}_{2 \rho_{2}}-L_{2 \rho_{2}} C_{2}$ is stable $\forall \rho_{21} \in \Theta_{\rho_{2}}$ by solving LMIs as in (22), where $\Theta_{\rho_{2}}$ is the hyper rectangle for $\rho_{21}$ which is defined in (2).

Remark 3: Due to Remark 1 and 2, one can see that all the varying parameters for LPV UI observers designed in III-A and III-B can be measured.

Remark 4: As we can see the system matrices in (33) and (43), the state repeats exactly the output of the system. It means that we use the information of the measured outputs to estimate itself. This observer helps to recover the real state of the system efficiently in case of disturbed outputs. Furthermore, this observer allows us to estimate the unknown input that affects the system. The information about unknown inputs is really useful for improving the quality of quadcopter control.

\section{Simulation RESUlts}

The quadcopter parameters for simulation are listed in the following table (Fig. I).

TABLE I

QUADCOPTER PARAMETERS DEFINITION

\begin{tabular}{|l|l|l|l|}
\hline Parameter & Name & Value & Unit \\
\hline$m$ & Quadcopter mass & 2.0 & $\mathrm{Kg}$ \\
\hline$l$ & Arm length & 0.23 & $\mathrm{~m}$ \\
\hline$b$ & Thrust coefficient & $7.73213 \times 10^{-6}$ & $\mathrm{~N} \cdot \mathrm{s}^{2}$ \\
\hline$d$ & Drag coefficient & $1.27513 \times 10^{-7}$ & $\mathrm{~N} \cdot \mathrm{m} \cdot \mathrm{s}^{2}$ \\
\hline$I_{x}, I_{y}$ & Inertia on $x$ and $y$ axis & 0.0142 & $\mathrm{Kg} \cdot \mathrm{m}^{2}$ \\
\hline$I_{z}$ & Inertia on $z$ axis & 0.0267 & $\mathrm{Kg} \cdot \mathrm{m}^{2}$ \\
\hline$J_{r}$ & Rotor inertia & $8.5 \times 10^{-4}$ & $\mathrm{Kg} \cdot \mathrm{m}^{2}$ \\
\hline$\omega_{i}$ & Rotor speed & {$[0,500]$} & $\mathrm{rad} \cdot \mathrm{s}^{-1}$ \\
\hline
\end{tabular}

Based on quadcopter's parameters in table I, and the definition of varying parameters in subsections III-A and III$\mathrm{B}$, the ranges of varying parameters are shown in the table II.

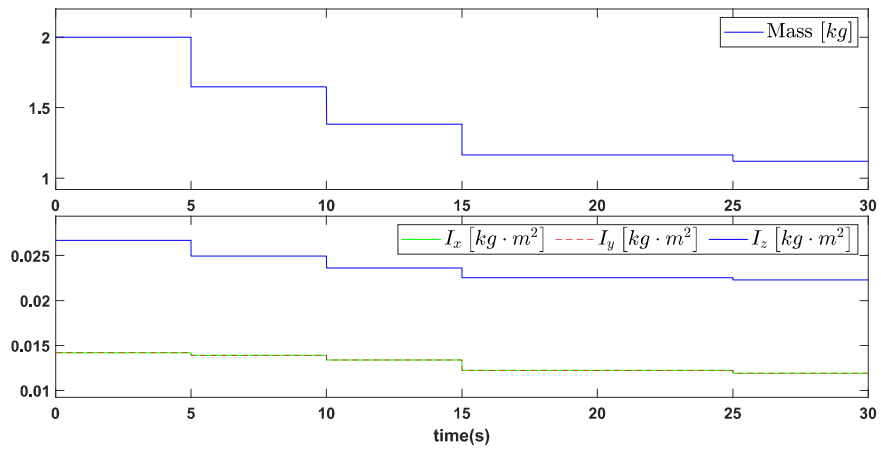

Fig. 3. Variations of mass and moments of inertia 

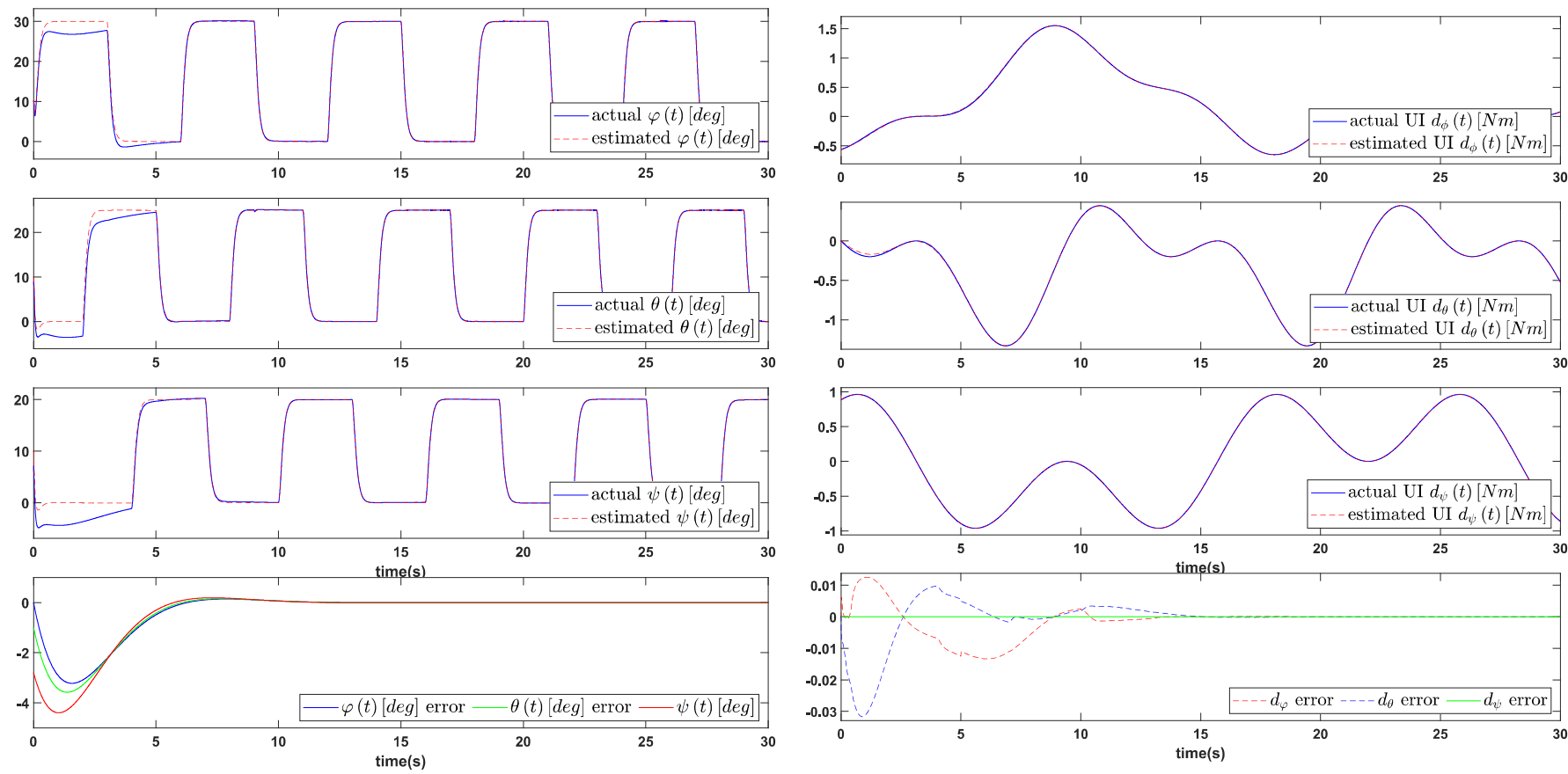

Fig. 4. States $\varphi, \theta, \psi$ vs estimated states $\varphi, \theta, \psi$

Fig. 6. Unknown Input estimation
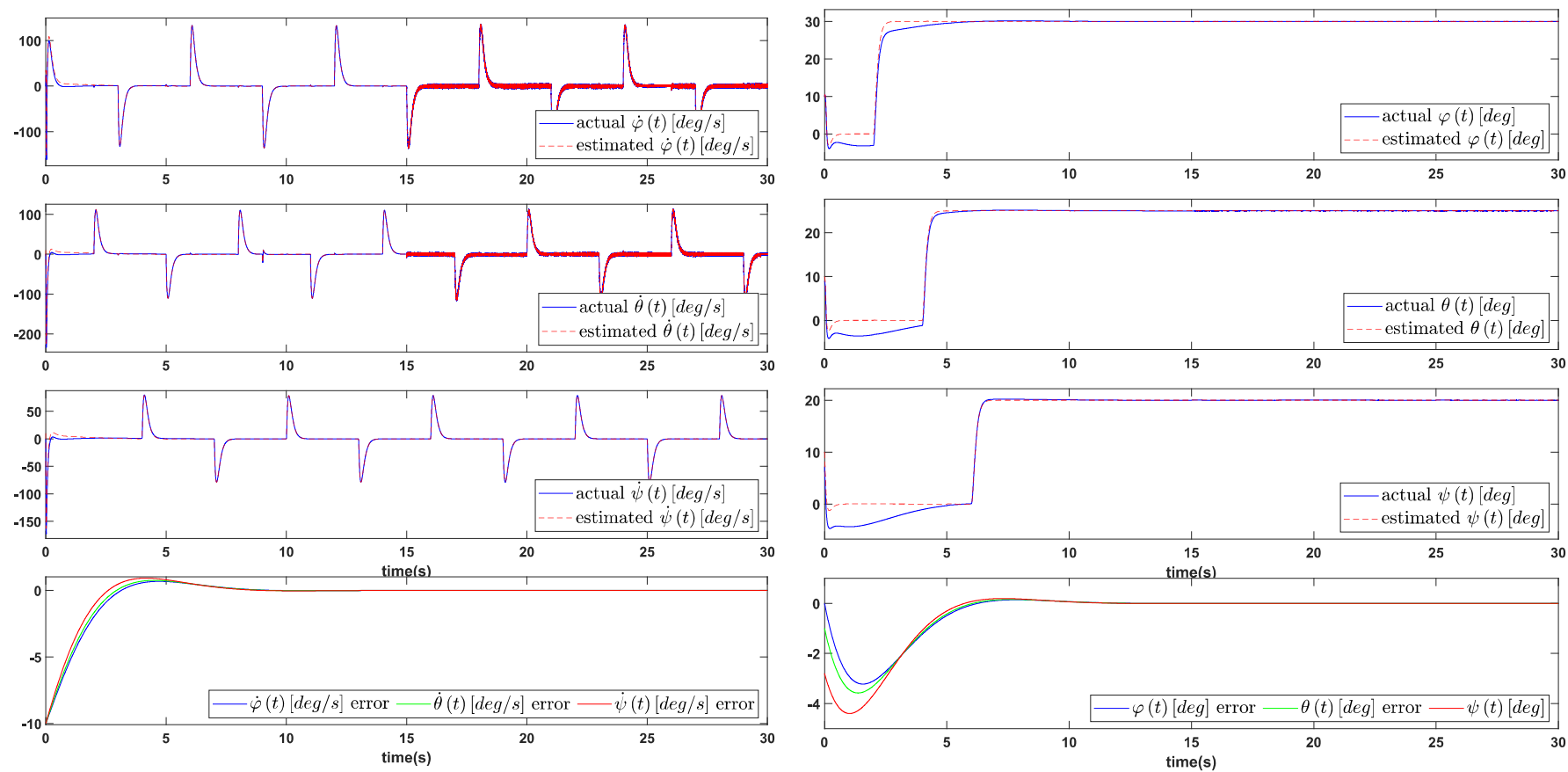

Fig. 5. States $\dot{\varphi}, \dot{\theta}, \dot{\psi}$ vs estimated states $\dot{\varphi}, \dot{\theta}, \dot{\psi}$

Fig. 7. States $\varphi, \theta, \psi$ vs estimated states $\varphi, \theta, \psi$

TABLE II

VARIATION RANGES OF VARYING PARAMETERS

\begin{tabular}{|c|c|c|}
\cline { 2 - 3 } \multicolumn{1}{c|}{} & $\underline{\rho}_{i}$ & $\bar{\rho}_{i}$ \\
\hline$\rho_{11}$ & 47.09580 & 84.0336 \\
\hline$\rho_{12}$ & 35.84230 & 44.8430 \\
\hline$\rho_{21}$ & -74.1176 & 74.1176 \\
\hline
\end{tabular}

For simulation, the mass of quadcopter is varying abruptly

between $5 s$ and $25 s$ from $2(\mathrm{~kg})$ to $1.12(\mathrm{~kg})$. Along with the quadcopter's mass variation, the moments of inertia $I_{x}, I_{y}, I_{z}$ around the three axes $I_{x}=I_{y} \in\left[\begin{array}{ll}0.0119 & 0.0142\end{array}\right]\left[\mathrm{kg} \cdot \mathrm{m}^{2}\right]$, and $I_{z} \in\left[\begin{array}{ll}0.0223 & 0.0267\end{array}\right]\left[\mathrm{kg} \cdot \mathrm{m}^{2}\right]$ abruptly change as in Fig. 3 .

The actual and estimated states of the quadcopter for the simulation with the impulse reference trajectories of $\varphi, \theta$, and $\psi$ are shown in Fig. 4 and Fig. 5. While the actual 

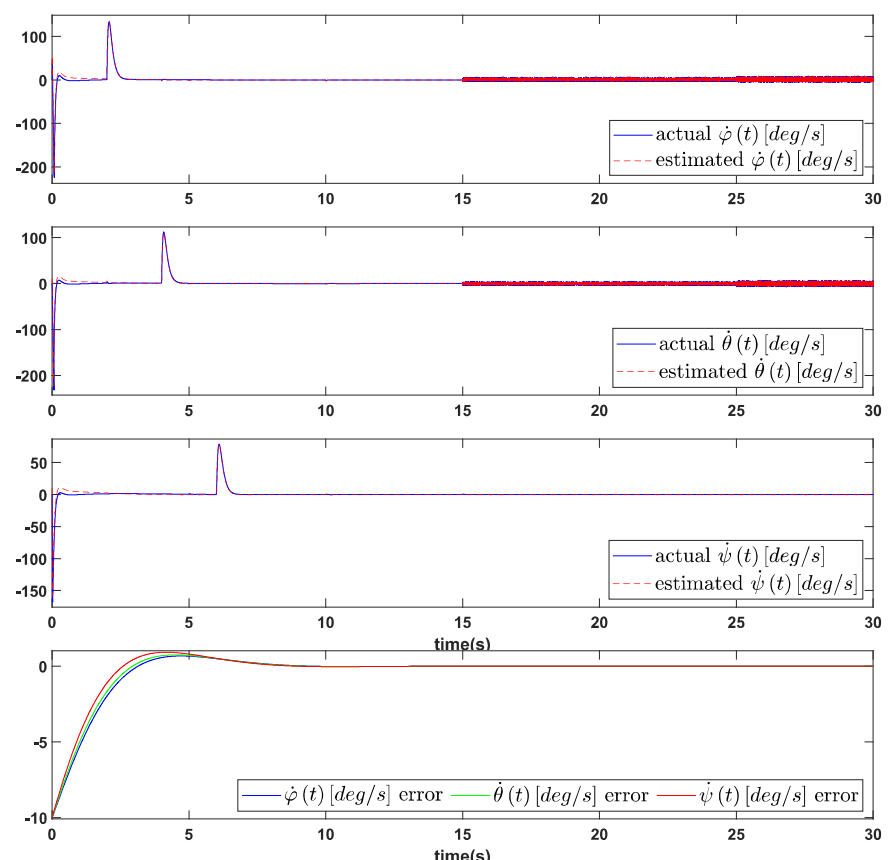

Fig. 8. States $\dot{\varphi}, \dot{\theta}, \dot{\psi}$ vs estimated states $\dot{\varphi}, \dot{\theta}, \dot{\psi}$
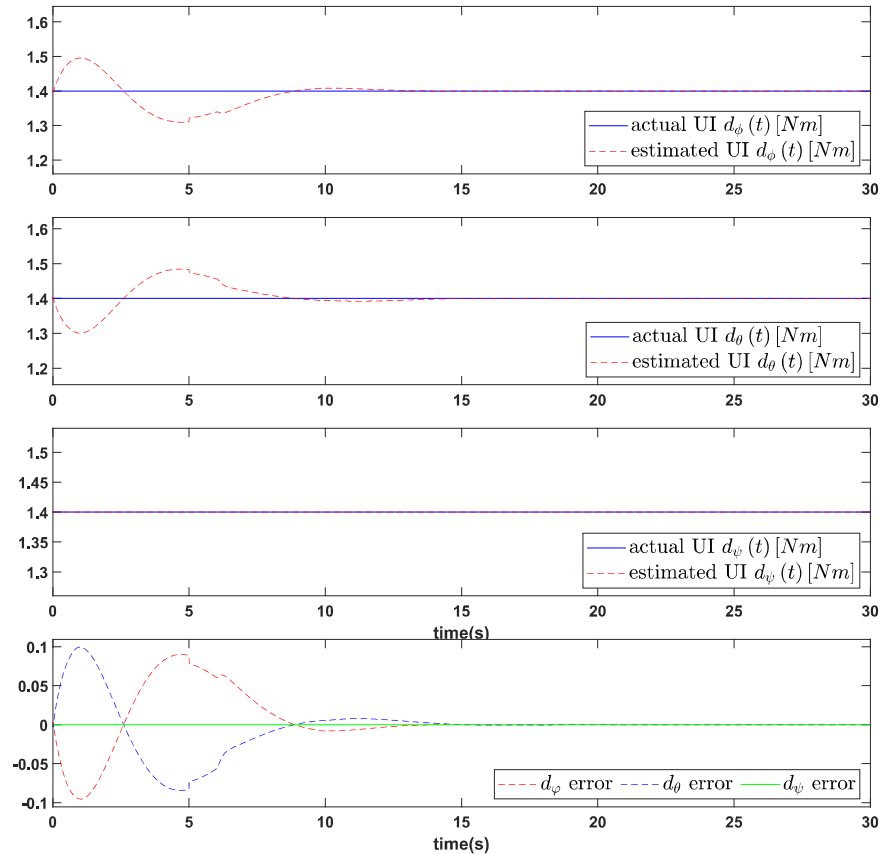

Fig. 9. Unknown Input estimation

and estimated disturbances (random disturbances) and their differences are shown in Fig. 6. The estimated state and disturbances converge to the actual values in about $7 \mathrm{~s}$.

In Fig. 7, 8, and 9 show the results for the case of step reference signals and constant disturbances.

Different simulations using multiple reference (impulse, step, random, and sin) and different types of disturbances (impulse, step, random, and sin) have shown a satisfactory performance of the proposed UIO.

The same simulations for slow variation of mass are also conducted, the state and unknown inputs are also well estimated.

\section{CONCLUSION AND FURTHER RESEARCH}

In this paper, a Linear Parameter Varying Unknown Input Observer for the attitude subsystem of a mass-varying quadcopter is proposed. By using algebraic matrix manipulation under the conditions stated in Theorem 1, the convergence of the UIO is guaranteed.

Further researches concern the design of an Unknown Input Observer-based controller for mass-varying quadcopter.

\section{REFERENCES}

[1] J. Chen and P. R. J, Robust Model-Based Fault Diagnosis for Dynamic Systems. Springer, 1999.

[2] R. Patton, R. Clark, and P. M. Frank, Fault Diagnosis in Dynamic Systems: Theory and Application. Prentice-Hall, 1989.

[3] J. El-Osery, Aly; Prevost, [Studies in Systems, Decision and Control] Control and Systems Engineering Volume 27 II Proportional-Integral Observer in Robust Control, Fault Detection, and Decentralized Control of Dynamic Systems, 2015, vol. 10.1007/978-3-319-14636-2, pp. 13-43.

[4] D. Luenberger, "An introduction to observers," IEEE Transactions on Automatic Control, vol. 16, no. 6, pp. 596 - 602, 1971.

[5] M. Darouach, M. Zasadzinski, and S. J. Xu, "Full-order observers for linear systems with unknown inputs," IEEE Transactions on Automatic Control, vol. 39, no. 3, pp. 606-609, 1994.

[6] I. N. Doye, M. Darouach, H. Voos, and M. Zasadzinski, "Design of unknown input fractional-order observers for fractional-order systems," International Journal of Applied Mathematics and Computer Science, vol. 23, no. 23, pp. 491-500, 2013.

[7] V. T. Kaczorec, "Proportional-integra lobserver sfo rlinea rmultivariable time-varyin gsystem s," Regelungstechnik, vol. 27, pp. 359-362, 1979.

[8] B. Wojciechowski, "Analysis and synthesis of proportional-integral observers for single-input-single-output time-invariant continuous systems," Ph.D. dissertation, The school of the thesis, The address of the publisher, 7 1978, an optional note.

[9] V. K. Tripathi, L. Behera, and N. Verma, "Disturbance observer based backstepping controller for a quadcopter," in IECON 2016 - 42nd Annual Conference of the IEEE Industrial Electronics Society, 2016, pp. 108-113.

[10] N. Ahmed and M. Chen, "Sliding mode control for quadrotor with disturbance observer," Advances in Mechanical Engineering, vol. 10, no. 7 , pp. 1-16, 2018.

[11] D. Ichalal and S. Mammar, "On unknown input observers for lpv systems," IEEE Transactions on Industrial Electronics, vol. 62, pp. $5870-5880,092015$.

[12] I. Alberto, Nonlinear Control Systems. Springer US, 1995, vol. 1.

[13] S. Bouabdallah, "Design and control of quadrotors with application to autonomous flying," 012007.

[14] S. Bouabdallah and R. Siegwart, "Backstepping and Sliding-mode Techniques Applied to an Indoor Micro Quadrotor," Intelligent Robots and Systems, 2007. IROS 2007. IEEE/RSJ International Conference, pp. $153-158,2007$.

[15] V. Wuest, V. Kumar, and G. Loianno, "Online estimation of geometric and inertia parameters for multirotor aerial vehicles," 2019 International Conference on Robotics and Automation (ICRA), vol. 20-24 May 2019, Montreal, QC, Canada, Canada, pp. 1884 - 1890, 2019. 